\title{
Cystic echinococcosis in cattle dairy farms: spatial distribution and epidemiological dynamics
}

\author{
Antonio Scala, ${ }^{1,2}$ Antonio Bosco, ${ }^{1,3}$ Anna Paola Pipia, ${ }^{1,2}$ Claudia Tamponi, $, 1,2$ \\ Vincenzo Musella, ${ }^{1,4}$ Nicola Costanzo, ${ }^{4}$ Francesco Testoni, ${ }^{2}$ Antonio Montisci, ${ }^{5}$ Giovanni Mocci, ${ }^{5}$ \\ Alessandro Longhi, ${ }^{5}$ Laura Tilocea, ${ }^{6}$ Laura Rinaldi, ${ }^{1,3}$ Giuseppe Cringoli, ${ }^{1,3}$ Antonio Varcasia ${ }^{1,2}$ \\ ${ }^{1}$ Inter-University Center for Research in Parasitology, Naples; ${ }^{2}$ Laboratory of Parasitology, Veterinary \\ Teaching Hospital, Department of Veterinary Medicine, University of Sassari, Sassari; \\ ${ }^{3}$ Department of Veterinary Medicine and Animal Productions, University of Naples Federico II, Naples; \\ ${ }^{4}$ Department of Health Sciences, Magna Gracia University, Catanzaro; ${ }^{5}$ Local Health Unit n.5, Oristano; \\ ${ }^{6}$ Private veterinary practitioner, Sassari, Italy
}

\begin{abstract}
A study monitoring cystic echinococcosis (CE) in adult dairy cattle from intensive livestock farms located in the municipality of Arborea (Sardinia, Italy) was carried out between 2012 and 2015. A retrospective study of veterinary reporting forms of post-mortem inspections in 10 different Italian slaughterhouses was also performed. In addition, data on viability and molecular characterisation of hydatid cysts removed from parasitised organs in cattle was carried out. A geographical information system (GIS) with data layers of the study area and the geo-referenced points of 160 cattle farms was constructed. CE was found in $21.9 \%(35 / 160)$ of the surveyed farms. The retrospective study revealed that $0.05 \%(13 / 23,656)$ of adult slaughtered animals (over one year of age) from Arborea had
\end{abstract}

Correspondence: Laura Rinaldi, Department of Veterinary Medicine and Animal Productions, University of Naples Federico II, via Delpino 1, 80137 Naples, Italy.

Tel: +39.081.2536281 - Fax: +39.081.2536282.

E-mail: 1rinaldi@unina.it

Key words: Cystic echinococcosis; Intensive farming; Dairy cattle; Geographical information systems; Sardinia.

Acknowledgements: the authors thank Mr. F. Salis, Technician, Parasitology Laboratory, University of Sassari, Italy for technical assistance during the study. We also thank Mrs. Giustina Casu (University of Sassari, Italy) for her linguistic revision of the manuscript.

Conflict of interest: the authors declare no potential conflict of interest.

Received for publication: 28 February 2017.

Revision received: 14 April 2017.

Accepted for publication: 16 April 2017.

(C) Copyright A. Scala et al., 2017

Licensee PAGEPress, Italy

Geospatial Health 2017; 12:562

doi:10.4081/gh.2017.562

This article is distributed under the terms of the Creative Commons Attribution Noncommercial License (CC BY-NC 4.0) which permits any noncommercial use, distribution, and reproduction in any medium, provided the original author(s) and source are credited. tested positive to $\mathrm{CE}$. The results stratified per year showed the following CE prevalences: $0.09 \%(5 / 5673)$ in $2012 ; 0.02 \%(1 / 5682)$ in $2013 ; 0.08 \%(5 / 6261)$ in 2014 ; and $0.03 \%(2 / 6040)$ in 2015 ( $\chi^{2}$ with 3 degrees of freedom $=3.81 ; \mathrm{P}=0.282$ ). The E. granulosus sensu stricto (formerly called G1 or sheep strain) was detected in all cysts subjected to molecular analysis. The GIS analysis showed that CE is fairly resilient in the Arborea territory where most of cattle farms are located, while a small cluster of cases was found located in the southeastern part of Arborea, close to districts where sheep farms are situated. The present survey reports the presence of CE in Sardinian dairy cattle intensive farms and suggests that the parasitic pressure of $\mathrm{CE}$ in the island continues to be very strong.

\section{Introduction}

Cystic echinococcosis (CE) caused by Echinococcus granulosus sensu lato is a zoonotic disease of worldwide importance that is widespread in the whole Mediterranean region, from North Africa to Greece, Turkey, Balkan regions, Mediterranean France and Spain as well as southern Italy with the islands of Sicily and Sardinia (Varcasia et al., 2011; Deplazes et al., 2017). It has been defined one of the most important parasitic zoonoses in several countries of the Mediterranean Basin. Small ruminants are the predominant livestock of Sardinia, with approximately 3 million sheep, representing two thirds of all sheep in Italy. Risk factors associated with transmission of CE in Sardinia are: i) the high number of the dog definitive host $(150,000)$; ii) farm slaughtering of small ruminants; and iii) socio-economic and cultural conditions (i.e. the low price of adult sheep, the scarce knowledge of zoonotic importance of CE by the farmers, etc.), which all contribute to the transmission of $E$. granulosus leading to a high rate of infection, particularly in small ruminants (Scala et al., 2006; Varcasia et al., 2011). Several papers have been published on CE in Sardinia, as this island represent a good epidemiological model (Conchedda et al., 2010; Kinkar et al., 2016; Scala et al., 2006; Tamarozzi et al., 2015; Varcasia et al., 2004, 2011). However, the role of cattle in the epidemiology and transmission of CE has always been questioned, as these large ruminants are often found be infected with non-host-specific Echinococcus species. Indeed, almost all genotypes/species of $E$. granulosus have been found in the island, except $E$. ortleppi, i.e. the G5 cattle strain (Varcasia et al., 2006, 2007; Busi et al., 2007). Prevalence values ranging between 19.6 and $41.5 \%$ have been 
found in cattle raised at pasture with almost degenerated hydatid cysts and low fertility rates, generally between 0.76 and 2.6\% (Scala et al., 2004; Varcasia et al., 2006). The incidental finding of CE in dairy cattle bred in intensive farms without access to pasture in the municipality of Arborea (in the central-western part of Sardinia, Italy) led us to carry out the present study to assess the spatial distribution and epidemiological dynamics of this zoonotic metacestodosis in cattle in Sardinia where CE is endemic.

\section{Materials and Methods}

\section{Study area and sampling}

The survey was carried out between 2012 and 2015 monitoring $\mathrm{CE}$ in adult dairy cattle from 160 intensive farms of the municipality of Arborea (Sardinia, Italy, 3946'21.94"N; 8³4'52.64"E). The study area has an extension of $100 \mathrm{~km}^{2}$, sheep farming is almost absent and dairy cattle are usually raised indoor and fed with stored fodder and pellets. Data were acquired on a total of 23,656 animals slaughtered at the abattoir in Arborea. In addition, a retrospective study for the years 2012-2015 was carried out examining the reporting forms received by the official veterinary department in Oristano (Sardinia, Italy). These forms reported the outcomes of post mortem inspection visits performed in 9 slaughterhouses located in different Italian regions where cattle from the municipality of Arborea were slaughtered.

\section{Fertility evaluation and molecular characterisation of cysts}

Hydatid cysts were removed from the parasitised organs (liver and lungs), counted and ranked as fertile or sterile. Fertility was assessed by determining protoscolex viability by microscopic examination, observing protoscoleces and their flame cells movements as described elsewhere (Varcasia et al., 2006). Molecular identification was carried out by polymerase chain reaction (PCR), by amplifying fragments within 2 mitochondrial genes, NADH dehydrogenase 1 (ND1) and cytochrome $\mathrm{C}$ oxidase subunit 1 ( $\operatorname{cox} 1)$, using DNA extracted from the cysts. First a semi-nested PCR protocol by Dinkel et al. (2004) was used for initial rapid screening of all samples to discriminate the G1/G2/G3 strain cluster of E. granulosus from the G5 and G6/7 strains. Molecular analyses were confirmed by sequencing the mitochondrial genes ND1 and cox1, as described by Bowles et al. (1992) and Bowles and McManus (1993). Nucleotide sequences obtained were compared to those available in GenBank ${ }^{\circledR}$ through the use of the basic local alignment search tool software BLAST ${ }^{\circledR}$ (National Center for Biotechnology Information, U.S. National Library of Medicine, Bethesda, MD, USA) and Mega 6.0 (Tamura et al., 2013).

\section{Geo-referencing of cattle and sheep farms}

A geographical information system (GIS) was constructed with the administrative data layers of the study area (Arborea and surrounding municipalities), the geo-referenced points of the 160 cattle farms under investigation and the total number of sheep farms located in the study area. For this purpose, the software ArcGIS (version 10.3, ESRI, Redlands, CA, USA) was used and the farm coordinates were available from the Italian National Database (Veterinary Information Systems; http://www.vetinfo.sanita.it) (Figure 1).

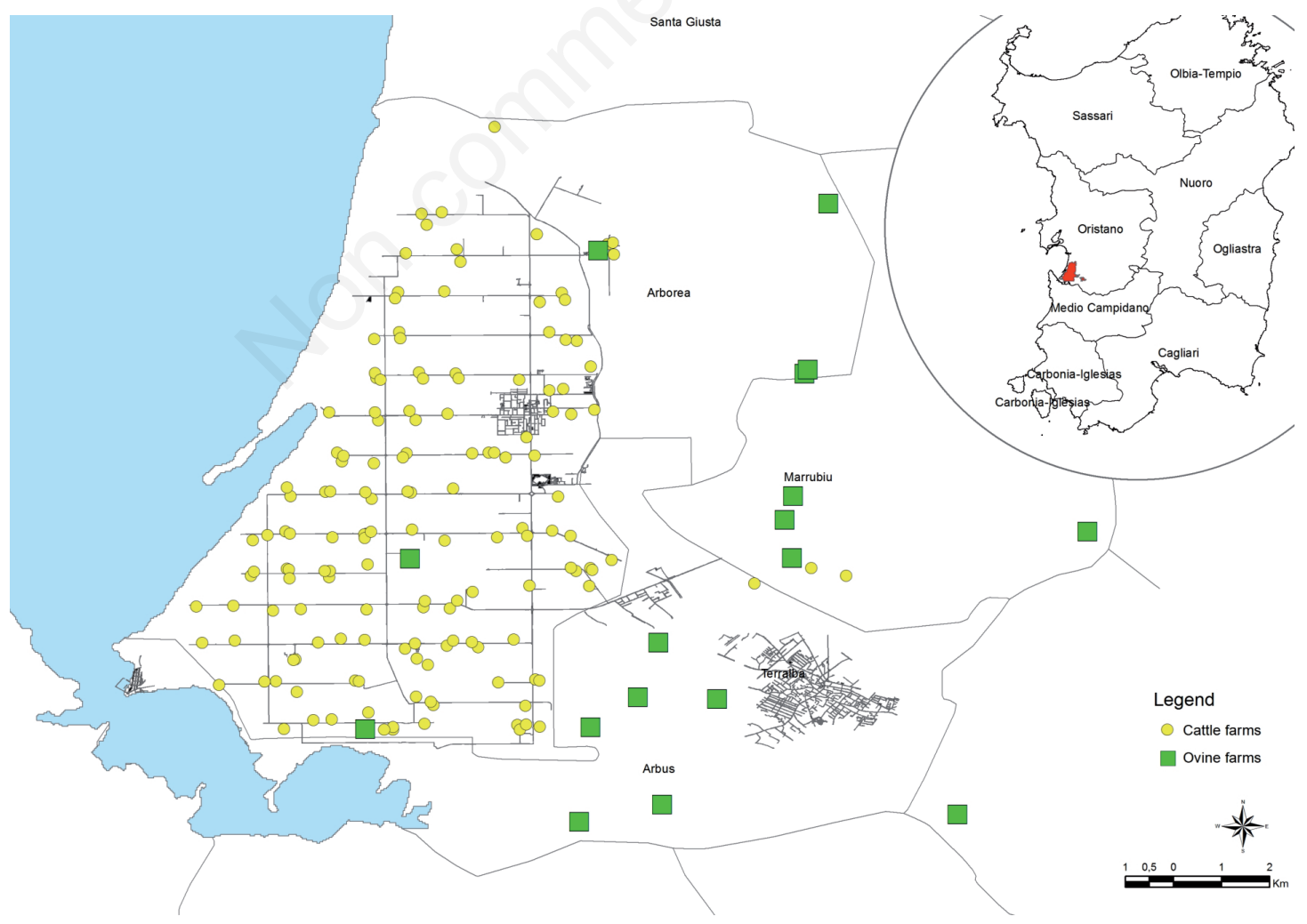

Figure 1. Point map showing the distribution of cattle and sheep farms in the study area. 


\section{Geographical information system analysis}

In order to display the spatial distribution of $\mathrm{CE}$ in cattle farms (used as epidemiological units in our study), as well as the spatial distribution of sheep farms located in the study area, a point map was drawn up within the GIS (Figure 2). Furthermore, for each cattle farm, the distance (minimum, average and maximum) from all the sheep farms in the study area was calculated (Cringoli et al., 2007). The clustering of CE positive farms was investigated based on location determined by exact coordinates and using the tool Average Nearest Neighbor Procedure in ArcGIS. The latter approach measures the distance between each feature centroid and its nearest neighbour centroid location, then averages all the nearest neighbour distances. The non-parametric Mann-Whitney U test (Mann and Whitney, 1947) was used to test differences among the above averaged value distances from sheep farms in CE positive and negative cattle farms. The statistical analysis was performed using SPSS 20.0 (IBM, Armonk, NY, USA).

\section{Results}

Cystic echinococcosis was found in 21.9\% (35/160) of the surveyed cattle farms. Thirteen out of 23,656 adult animals examined were found to be infected by $\mathrm{CE}(0.05 \%)$. Positive reports stratified per year showed the following rates: $0.09 \%(5 / 5673)$ in 2012 ; $0.02 \%(1 / 5682)$ in $2013 ; 0.08 \%(5 / 6261)$ in 2014 ; and $0.03 \%$ $(2 / 6040)$ in 2015 ( $\chi^{2}$ with 3 degrees of freedom $\left.=3.81 ; \mathrm{P}=0.282\right)$. Seventy-two veterinary reporting forms reported positive CE tests in 10 slaughterhouses located in different Italian regions. In particular, from 52 veterinary reporting forms, it was possible also to retrieve data about hydatid cyst distribution in the liver and lungs. In positive cattle, the metacestode was found in the liver in $71.2 \%$ (37/52) of cases, in 55.8\% (29/52) in the lungs and in $1.9 \%(1 / 52)$ in the kidneys. No statistically significant difference was observed between the prevalence rates found in the liver and in the lungs $\left(\chi^{2}=2.65 ; \mathrm{P}=0.103\right)$. The simultaneous presence of hydatid cysts in livers and lungs was found in $26.9 \%(14 / 52)$ of the positive animals; one cattle harboured cysts also in the kidney $(1.9 \%)$.

No viable cyst was found among those examined (100\% sterile). Cysts from a subsample of 13 cattle slaughtered in Arborea were used to perform molecular analysis. Rapid PCR screening demonstrated that all isolates belonged to the G1 genotype, i.e. E. granulosus sensu stricto (also called sheep strain). This diagnosis was confirmed by sequencing the mitochondrial ND1 and coxl genes; the sequences obtained were analysed and compared to those reported in GenBank ${ }^{\circledR}$ (https://www.ncbi.nlm.nih.gov/genbank/).

The cluster analysis performed by GIS showed a homogeneous distribution of CE positive cattle farms across the entire study area with a small cluster in the southeastern part of the study area (Figure 3). Specifically, the cluster/outlier type (COType) field distinguished between a statistically significant cluster of high values $(\mathrm{HH})$, of low values (LL), an outlier in which a high value was surrounded primarily by low values (HL) and an outlier in which a low value was surrounded primarily by high values (LH) as described by Mitchell (2005).

Table 1 summarises the data regarding the distance between

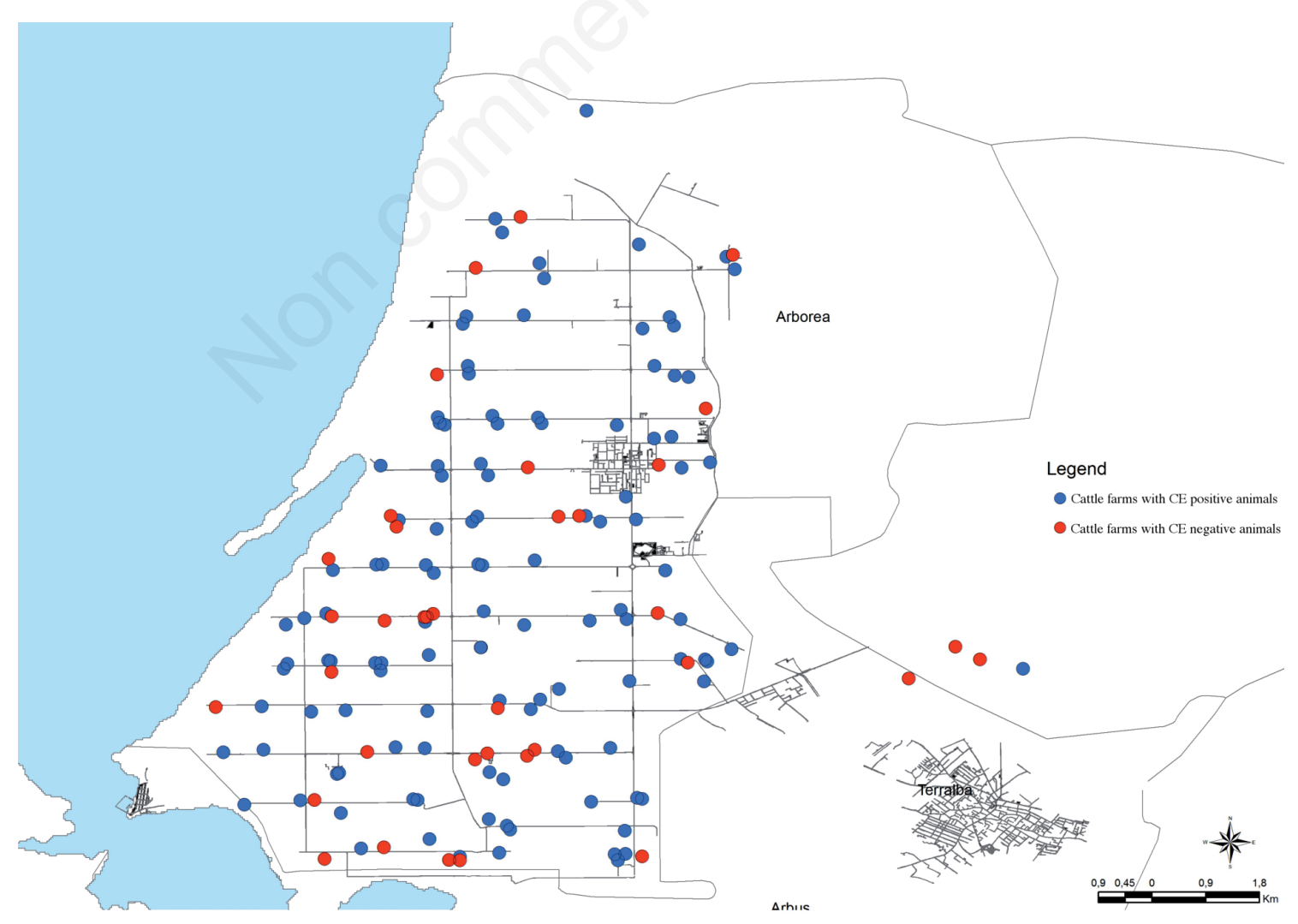

Figure 2. Distribution of cattle farms with cystic echinococcosis positive or negative animals. 
the sheep farms in the study area and the cattle farms tested for CE (positive and negative). Overall, the mean distances around the cattle farms positive to $\mathrm{CE}$ were significantly lower $(\mathrm{P}<0.001)$ than those around the cattle farms negative to $\mathrm{CE}$.

\section{Discussion}

The present survey reports for the first time the presence of CE in Sardinian dairy cattle bred with intensive farming system. The dairy cattle monitored in the Arborea area comprise a $100-\mathrm{km}^{2}$ geographical enclave with no access to pasture, no direct contact with shepherd dogs and/or sheep farms. The findings of the present study showed the presence of cattle positive to CE in $21.9 \%$ of the farms in the area, thus confirming that the parasitic pressure of $\mathrm{CE}$ in Sardinia continues to be strong despite several sanitary information initiatives carried out in the past (Varcasia et al., 2011).

The species isolated with the molecular analysis was E. granulosus sensu stricto (formerly called G1 or sheep strain) and no fertile cysts were found during our investigations. Taken together, these data suggest that there is no evidence of a cattle/dog lifecycle and that an external contamination likely occurred in the cattle farms. Moreover, illegal home slaughtering of animals, which is still a very common practice in sheep farms in Sardinia (Varcasia et al., 2011), is not performed in intensive dairy cattle farming in Arborea.

Different hypotheses were advanced to explain the spatial distribution, epidemiological dynamics and route of transmission of $\mathrm{CE}$ to dairy cattle bred in intensive farming system in Sardinia. The first consideration must be that the trend of CE infection in cattle has been more or less constant over the years examined, which means that the factors determining the persistence of $\mathrm{CE}$ in this area cannot be due to an occasional introduction of this infection. An incursion of E. granulosus eggs through forage seems a remote possibility; however, some authors have suggested it as a possible route of transmission of other metacestodoses, such as cysticercosis by Taenia hydatigena in goats from northern Italy (Manfredi et al., 2006). The majority of Arborea farms use their own forage and hay and silage are usually stored after sun drying, which means that the internal temperature increase of this forage by normal fermentation should not allow long-time survival of oncospheres. This opinion is supported by Nosik (1952), who showed loss of vitality of E. granulosus eggs after 1 hour at $50^{\circ} \mathrm{C}$ or 20 seconds at $100^{\circ} \mathrm{C}$. Similar results were also obtained by Meymarian (1961) and more recently by Thevenet et al. (2005). Although farfetched, and to some extent refuted by the constant infection trend, the hypothesis by Torgerson et al. (1995) that the occurrence of CE (and that of other metacestodoses) be due to egg dispersion by birds and coprophagous insects, cannot be excluded. Still, the most likely way of contamination might be through the access to the cattle farms of stray dogs, a common problem in some areas of Sardinia and elsewhere (Cringoli et al., 2007; Varcasia et al., 2011; Deplazes et al., 2017).

Stray dogs most probably enter farms for feeding on discarded placentas from cattle immediately after birth, especially during

Table 1. Mean distance of the sheep farms from the cattle farms positive and negative to cystic echinococcosis in the study area (Arborea, Sardinia, Italy).

\begin{tabular}{lcccc} 
Cattle farms & Distances of sheep farms from cattle farms \\
& Mean $(\mathrm{km})$ & SD & Min & Max \\
CE negative & 8.75 & 1.16 & 0 & 13.03 \\
CE positive & $7.29^{* *}$ & 0.31 & 0.03 & 16.73 \\
\hline
\end{tabular}

CE, cystic echinococcosis; SD, standard deviation. ${ }^{*}{ }^{*} \mathrm{P}<0.001$.
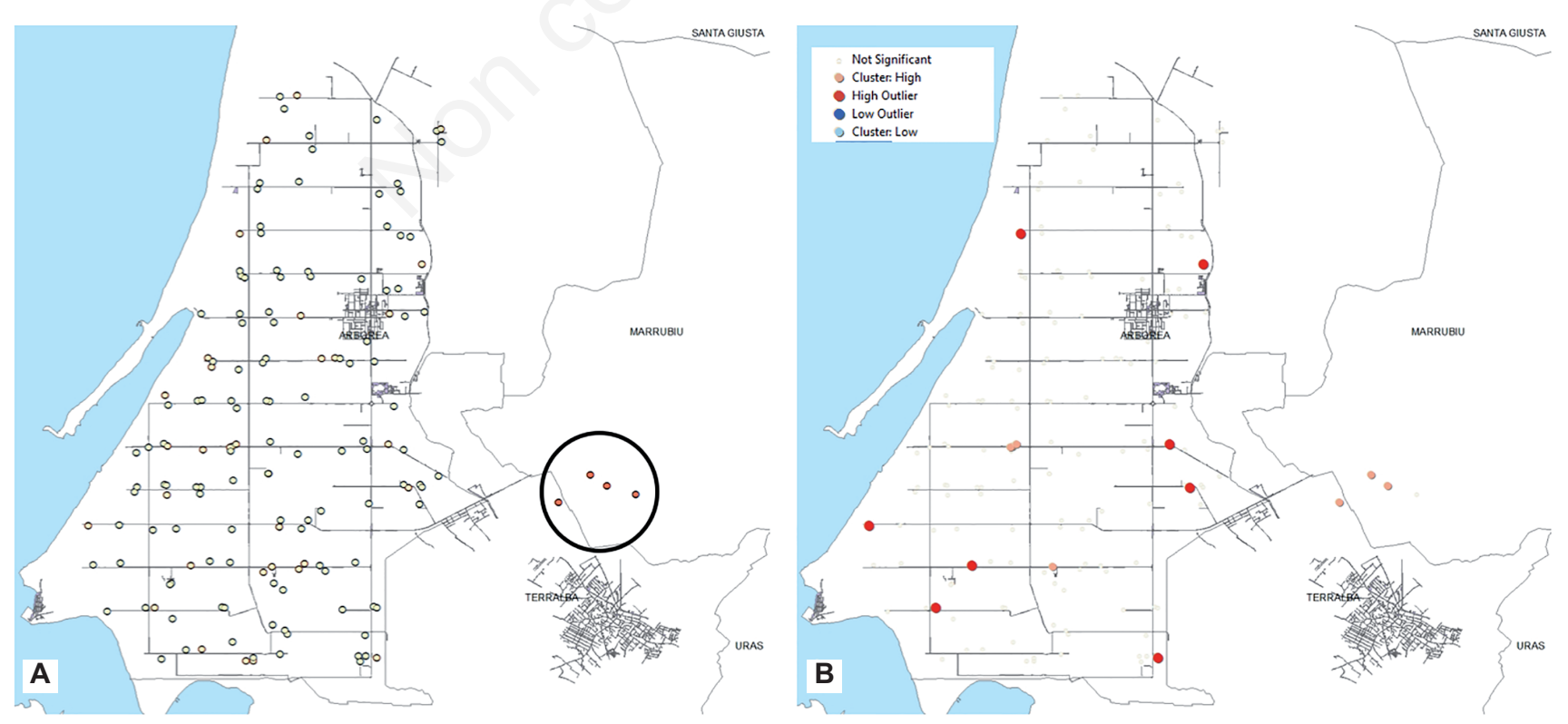

Figure 3. Clusters analysis of distribution of cattle farms positive to cystic echinococcosis: A) highlights the cluster area with a circle; B) shows the COType analysis. 
night. It is likely that these free-ranging dogs became infected by CE when feeding on CE-infected sheep carcasses in the sheep farms and then shed infectious eggs in proximate dairy cattle farms. This possible epidemiological dynamic is supported by the GIS analysis: the dairy cattle farms positive to CE were uniformly distributed in the study area, and only a small cluster of positive farms was detected in the south-eastern part of Arborea which is much closer to other zones of Sardinia where sheep farms are situated. In addition, the closer the sheep farms were to the cattle farms, the higher the risk of infection of the latter through stray dogs infected with E. granulosus by sheep offal ingestion. Noteworthy, GIS analysis showed that CE cases can be found also in the interior of Arborea Municipality, and breeders refer that packs of stray dogs circulate freely in the surrounding pine forests; additionally, dogs may feed from the same corn flour and barley used to feed the cattle, which open the prospect of dog faeces contaminating the cattle food; in fact, dogs faeces are often found in the very same mangers. Similarly, a study by Cringoli et al. (2007) showed by GIS analysis the predominant role of free-ranging dogs in the transmission cycle of $\mathrm{CE}$ in relation to cattle and water buffaloes.

With respect to the parasitological infection sites in liver and lungs, no significant difference in prevalence was found $(\mathrm{P}>0.05)$; these data are consistent with previous surveys (Varcasia et al., 2004; Poglayen et al., 2011).

\section{Conclusions}

The results of the present study confirm that GIS is a powerful descriptive analytical tool for the study of the spatial structure of animal populations and the epidemiological dynamics of CE transmission (e.g., stray dog movement within and between farms). Although contamination relying on a dog/cattle lifecycle seems unlikely, the presence of CE-positive dairy cattle in $22 \%$ of intensive farms in the study area testifies to a high environmental contamination by $E$. granulosus that must be taken into account (through dissemination activities, management of stray-dogs and control programmes) in order to prevent human infection risks.

\section{References}

Bowles J, Blair D, McManus DP, 1992. Genetic variants within the genus Echinococcus identified by mitochondrial DNA sequencing. Mol Biochem Parasit 54:165-74.

Bowles J, McManus DP, 1993. Molecular variation in Echinococcus. Acta Trop 53:291-305.

Busi M, Snabel V, Varcasia A, Garippa G, Perrone V, De Liberato C, D'Amelio S, 2007. Genetic variation within and between G1 and G3 genotypes of Echinococcus granulosus in Italy revealed by multilocus DNA sequencing. Vet Parasitol 150:7583.

Conchedda M, Antonelli A, Caddori A, Gabriele F, 2010. A retrospective analysis of human cystic echinococcosis in Sardinia (Italy), an endemic Mediterranean region, from 2001 to 2005. Parasitol Int 59:454-9.

Cringoli G, Rinaldi L, Musella V, Veneziano V, Maurelli MP, Di Pietro F, Frisiello M, Di Pietro S, 2007. Geo-referencing livestock farms as tool for studying cystic echinococcosis epidemi- ology in cattle and water buffaloes from southern Italy. Geospat Health 2:105-11.

Deplazes P, Rinaldi L, Alvarez Rojas CA, Torgerson PR, Harandi MF, Romig T, Antolova D, Schurer JM, Lahmar S, Cringoli G, Magambo J, Thompson RCA, Jenkins EG, 2017. Global distribution of alveolar and cystic echinococcosis. Adv Parasitol 95:315-493.

Dinkel A, Njoroge EM, Zimmermann A, Wälz M, Zeyhle E, Elmahdi IE, Mackenstedt U, Romig T, 2004. A PCR system for detection of species and genotypes of the Echinococcus granulosus complex, with reference to the epidemiological situation in eastern Africa. Int J Parasitol 34:645-53.

Kinkar L, Laurimäe T, Simsek S, Balkaya I, Casulli A, Manfredi Mt, Ponce-Gordo F, Varcasia A, Lavikainen A, González Lm, Rehbein S, Van Der Giessen J, Sprong H, Saarma U, 2016. High-resolution phylogeography of zoonotic tapeworm Echinococcus granulosus sensu stricto genotype G1 with anemphasis on its distribution in Turkey, Italy and Spain. Parasitol 143:1790-801.

Manfredi MT, Ghirardelli R, Zanzani S, 2006. Infestazione da Cysticercus tenuicollis in un allevamento di capre. Parassitologia 48:433-6.

Mann HB, Whitney DR, 1947. On a test of whether one of two random variables is stochastically larger than the other. Ann Math Statist 18:50-60.

Meymariam E, 1961. Host parasite relationships in echinococcosis. VI. Hatching and activation of Echinococcus granulosus ova in vitro. Am J Trop Med Hyg 10:719-26.

Mitchell A, 2005. The ESRI guide to GIS analysis, Volume 2. ESRI Press, Redlands, CA, USA.

Nosik AF, 1952. Resistence of oncospheres of Echinococcus granulosus to influence of some physical and chemical factors. Proc Kharkov Vet Inst 21:304-11.

Poglayen G, Varcasia A, Scala A, Pipia AP, Masi M, Marchesi B, Parigi M, 2011. Epidemiological and biomolecular survey on cystic echinococcosis of cattle in Italy. In: International Congress of Mediterannean Federation of Health and Production of Ruminants, 19, Belgrade (Serbia), 25-28 May 2011. University of Belgrade, Faculty of Veterinary Medicine, Belgrade, Serbia.

Scala A, Canu S, Tanda B, Basciu M, Polinas L, Sanna Coccone GN, Pilloni S, Canu S, Varcasia A, Garippa G, 2004. An epidemiological a biomolecular survey of cystic echinococcosis in cattle in Sardinia. Parassitologia 46:443-4.

Scala A, Garippa G, Varcasia A, Tranquillo VM, Genchi C, 2006. Cystic echinococcosis in slaughtered sheep in Sardinia (Italy). Vet Parasitol 15:33-8.

Tamarozzi F, Rossi P, Galati F, Mariconti M, Nicoletti GJ, Rinaldi F, Casulli A, Pozio E, Brunetti E, 2015. The Italian registry of cystic echinococcosis (RIEC): the first prospective registry with a European future. Euro Surveill 7:20.

Tamura K, Stecher G, Peterson D, Filipski A, Kumar S, 2013. MEGA6: molecular evolutionary genetics analysis version 6.0. Mol Biol Evol 30:2725-9.

Thevenet PS, Jensen O, Drut R, Cerrone GE, Grenóvero MS, Alvarez HM, Targovnik HM, Basualdo JA, 2005. Viability and infectiousness of eggs of Echinococcus granulosus aged under natural conditions of inferior arid climate. Vet Parasitol 133:71-7.

Torgerson PR, Pilkington J, Gulland FM, Gemmell MA, 1995. Further evidence for the long distance dispersal of taeniid 
eggs. Int J Parasitol 25:265-7.

Varcasia A, Canu S, Kogkos A, Pipia AP, Scala A, Garippa G, Seimenis A, 2007. Molecular characterization of Echinococcus granulosus in sheep and goats of Peloponnesus, Greece. Parasitol Res 101:1135-9.

Varcasia A, Canu S, Lightowlers MW, Scala A, Garippa G, 2006. Molecular characterization of Echinococcus granulosus strains in Sardinia. Parasitol Res 98:273-7.

Varcasia A, Garippa G, Scala A, 2004. The diagnosis of Echinococcus granulosus in dogs. Parassitologia 46:409-12.

Varcasia A, Tanda B, Giobbe M, Solinas C, Pipia AP, Malgor R, Carmona C, Garippa G, Scala A, 2011. Cystic echinococcosis in Sardinia: farmers' knowledge and dogs infection in sheep farms. Vet Parasitol 181:335-40. 